\section{Der Fahrsimulatortest „carsim“ zur Erfassung der Vigilanz. Einfluss der Fahrpraxis und weiterer Faktoren bei Gesunden und Patienten mit Schlafapnoe-Syndrom}

Zusammenfassung: Patienten mit obstruktiver Schlafapnoe (OSAS) leiden u.a. an einer Beeinträchtigung der Daueraufmerksamkeit. Zu deren Erfassung und Objektivierung sowie Therapieverlaufsbeobachtung sind Fahrsimulationsprogramme besonders geeignet. Das von uns neu entwickelte ComputerProgramm „carsim“ simuliert auf einem Bildschirm eine Straße mit Kurven, wobei das Fahrzeug durch Lenkradbewegungen (tracking) auf der rechten Spur gehalten werden muss. Neben der Untersuchung der Gütekriterien (Objektivität, Reliabilität, Validität) sowie der Normwerteerstellung ist auch der Einfluss von wichtigen Variablen (Alter, Geschlecht, Schulausbildung, Beruf, Störungsdauer, AHI, BMI, visuelle und motorische Funktionsfähigkeit, Fahrerlaubnis, Fahrpraxis, Epworth-Score, Unfallhäufigkeit) an Gesunden und Patienten zu überprüfen. Deshalb wurden 100 Gesunde, 200 OSAS-Patienten und 30 Patienten unter nCPAP analysiert. Bei gesunden Personen und Patienten unter nCPAP spielte die Fahrpraxis hinsichtlich der Ergebnisse in der Fahrsimulation keine Rolle. OSAS-Patienten ohne Führerschein sowie ohne Fahrpraxis erreichten schlechtere TrackingErgebnisse als Patienten mit Führerschein bzw. langer und häufiger Fahrpraxis (ohne Fahrerlaubnis: $\bar{x}=8058 \pm 10878$ Spurabweichungen; mit Fahrerlaubnis: $\bar{x}=2111 \pm 6564$ Spurabweichungen; $\mathrm{p}<, 001)$. Unbehandelte OSAS-Patienten sind demnach nicht nur in ihrer Aufmerksamkeitsleistung extrem beeinträchtigt, sondern auch in ihren Coping-Strategien.

The Driving Simulation Test “carsim” for Assessing Vigilance. Influence of Driving Practice and Other Factors on Healthy Subjects and on Sleep Apnoea Syndrome Patients: Among other factors, obstructive sleep apnoea (OSAS) patients suffer from impaired continuous attention. For registration and objectification as well as observation of the course of therapy, driving simulation programmes are particularly suitable. "Carsim", a driving simulation newly developed by us, simulates a bendy road via a screen where a vehicle is supposed to be kept on the right lane by operating a steering wheel. Apart from examination of quality criteria (objectivity, reliability, validity) and establishment of standard values, the influence of significant variables (age, gender, school education, profession, duration of disorder, AHI, BMI, visual and motor functionability, driving license, driving experience, Epworth score, accident frequency) on patients and healthy controls requires to be

Pneumologie 2000; 54: 338-344

(c) Georg Thieme Verlag Stuttgart · New York ISSN 0934-8387

\author{
A. Büttner ${ }^{1}$, W. Randerath ${ }^{1}$, K.-H. Rühle \\ ${ }^{1}$ Klinik Ambrock, Klinik für Pneumologie, Allergologie und \\ Schlafmedizin, Universität Witten-Herdecke, Hagen \\ (K.-H. Rühle)
}

evaluated. For this purpose, 100 healthy controls, 200 OSAS patients and 30 patients under nCPAP were analysed. In healthy persons and patients under nCPAP, driving experience had no impact on the driving simulation results. OSAS patients with no driving license and no driving experience achieved lower marks for tracking than patients with driving license or a lot of driving experience (no driving license: $\bar{x}=8058 \pm 10878$ track deviations; with driving licence: $\bar{x}=2111 \pm 6564$ track deviations; $\mathrm{p}<.001)$. According to our findings, untreated OSAS severely interferes with patients' attention capacity as well as their coping strategies.

Key words: Sleep apnoea syndrome - Reference values - Driving simulation - Continuous attention

\section{Einleitung}

Patienten mit obstruktiver Schlafapnoe leiden, neben ihrer eigentlichen Symptomatik, häufig an einer Vielzahl von Folgeerscheinungen, u.a. an exzessiver Tagesmüdigkeit und einer verminderten Daueraufmerksamkeit $[3,4,20,24,25,27,28,33$, 36]. Auslösend sind hierfür nächtliche Arousals mit konsekutiver Schlaffragmentierung [19,34,35]. Diese Leistungseinschränkung beeinflusst die Betroffenen sowohl beruflich als auch in ihrer Fähigkeit, Kraftfahrzeuge zu führen [2,9,10,11, $12,13,14,15,19,20,26,27,29,33,34,35,36,38,39]$. Folge dieser verminderten Leistungsfähigkeit sind daher häufig Unfälle oder Beinaheunfälle durch Einschlafen am Steuer [19,34,35, $38,39]$.

Aldrich et al. führten aufgrund seiner Untersuchungen $71 \%$ der Unfälle von 424 schlafgestörten Patienten auf die extreme Schläfrigkeit tagsüber zurück, wobei der Schweregrad der Erkrankung einen wesentlichen Einfluss besaß [1,38,39]. Laut George et al. lag sogar bei $93 \%$ der OSAS-Patienten ein erhöhtes Unfallrisiko vor $[9,18,38,39]$. Findley et al. errechneten ein drei- bis siebenfach erhöhtes $[9,11,38,39]$.

Unter nCPAP-Therapie ist bereits nach kurzer Zeit ein deutlicher Rückgang der Tagesmüdigkeit sichtbar, deren Erfassung zumeist mit dem Multiple-Sleep-Latency-Test MSLT [8] und den Maintenance of Wakefulness-Test MWT [32] erfolgte.

Während der MSLT die Einschlaflatenz in monotonen Situationen erfasst, wird mit dem MWT die Fähigkeit, wach zu bleiben, gemessen und objektiviert. Letzterer wird daher bei 
den verschiedensten klinischen Fragestellungen eingesetzt, insbesondere zur Beurteilung der Fahrtauglichkeit und spezieller beruflicher Anforderungen [19].

Da sowohl der MSLT als auch der MWT das Wachheitsstadium messen, untersuchte Walsleben, ob beide auch die Wachsamkeit („alertness“) erfassen bzw. ob das gemessene Wachheitsstadium der Wachsamkeit entspricht. Da dies nicht der Fall ist, mussten Untersuchungsverfahren gesucht werden, die die Daueraufmerksamkeit bzw. die Vigilanz angemessener untersuchen [34,37].

Seit einiger Zeit werden daher verstärkt Fahrsimulationsprogramme eingesetzt, um Vigilanz und Daueraufmerksamkeitsdefizite bei Patienten mit obstruktiver Schlafapnoe zu erfassen [d. Verf.].

So stellte Findleys Arbeitsgruppe 1989 einen Fahrsimulator (Steer Clear) zur Erfassung der Fahrtauglichkeit von OSASPatienten vor, bei dem eine monotone Fahrsituation dargestellt wird und Hindernisse umfahren werden müssen [12]. Findley et al. konnten nachweisen, dass Patienten mit obstruktiver Schlafapnoe deutlich schlechtere Testergebnisse (ca. dreifache Fehlerrate) erreichten als die Personen der Kontrollgruppe und dass ungünstige Testergebnisse Korrelationen mit einer hohen Unfallrate ergaben $[9,11,12,13,14$, 19,34]. Bei einer Untersuchung vor und nach nCPAP-Therapie konnte beim Steer Clear eine deutliche Besserung der Fehlerrate (50\%ige Reduktion) nach 3 - bis 5-monatiger Therapie festgestellt werden $[12,19,34]$.

In der Folge wurden verschiedene Fahrsimulatoren, u.a. der DADT (Divided-Attention-Driving-Test) von George et al. entwickelt, die den multimodalen Aufbau der komplexen Autofahrleistung erfassen sollten $[16,17,18,19,34]$. Der DADT beruht auf Moscowitz et al. Test zur geteilten Aufmerksamkeit [30]. Per tracking soll ein Positionsindikator in einer Zielbox gehalten werden $[16,17]$. Die entscheidenden Komponenten sind hierbei das Steuern (tracking) und das visuelle Absuchen der Umgebung nach relevanten Reizen (visual search) $[16,17,18]$. Cassel et al. benutzten hingegen einen 80minütigen Daueraufmerksamkeitstest, um nCPAP-Effekte nachzuweisen [10].

In unserer Arbeitsgruppe wurde 1997 ebenfalls ein Fahrsimulatortest (Ambrocker Vigilanztest) entwickelt, der den Vigilanzgrad erfassen soll. Er beruht auf den Prinzipien des Steer Clear von Findley et al. [12] und des DADT von George et al. $[16,17,18]$. Auch bei ihm fand sich prätherapeutisch eine wesentlich höhere Fehlerrate als unter nCPAP-Therapie und gegenüber einer Kontrollgruppe [19,34,35].

Der von uns entwickelte und validierte Fahrsimulator „carsim“ basiert auf dem oben beschriebenen Prinzipien, ist aber eine konsequente Weiterentwicklung. Während George et al. nur einen Positionsindikator (Abb. 1) verwenden, der in einer beweglichen Zielbox gehalten werden soll, wird bei carsim (Abb. 2) auf einem Computer-Bildschirm eine Straße mit Kurven, Randbegrenzung und Mittelstreifen simuliert. Abhängig von den Lenkradbewegungen (tracking), wird die Position des Fahrzeugs auf der Straße neu berechnet und online visualisiert, wodurch eine realistische und interaktive Fahrsituation erzielt wird.

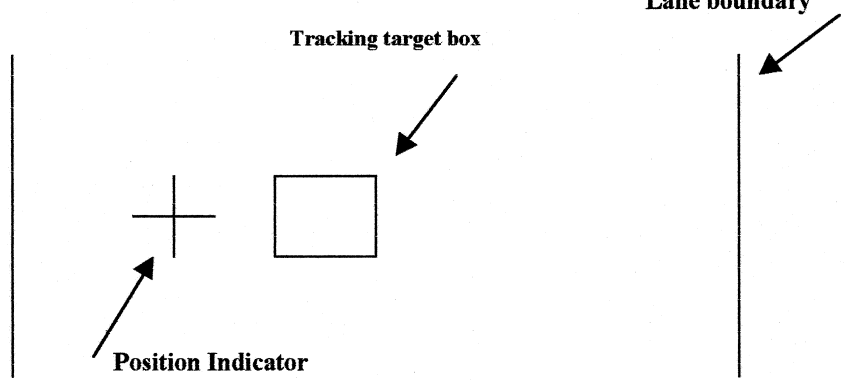

Abb. 1 DADT (Divided-Attention-Driving-Test) nach George et al. 1996a, 1996b.

Aufgrund der oben beschriebenen Problematik scheinen Fahrsimulationsprogramme die geeigneten Instrumente zum Screening und zur Therapieverlaufskontrolle bei OSAS-Patienten zu sein, da sie einerseits die Daueraufmerksamkeit global erfassen und andererseits auch die komplexe Leistung des Autofahrens simulieren.

Das Fahrsimulatorprogramm „carsim“ soll in der klinischen Routine, bei speziellen gutachterlichen Fragestellungen (bspw. Entzug des Führerscheins, Berentung) sowie bei beruflich gefährdeten Personen (u.a. Berufskraftfahrern) eingesetzt werden. $\mathrm{Zu}$ diesem Zweck wurde er von uns zunächst optimiert und validiert, außerdem mussten die Normwerte für die zwei wichtigsten Parameter (Spur- und Toleranzabweichungen; vgl. Tab. 1) anhand eines gesunden Vergleichskollektivs erhoben und seine Gültigkeit anhand einer umfangreichen Fragestellung im klinischen Bereich überprüft [7].

Tab. 1 Normwerte der Gesunden $(n=100)$

\begin{tabular}{lc}
\hline gemessene Parameter & Mittelwert \pm Standardabweichung \\
\hline Spurabweichungen & $23,16 \pm 44,73$ \\
Toleranzabweichungen & $960,73 \pm 1770,45$ \\
\hline
\end{tabular}

Besonders wichtig ist es in diesem Zusammenhang, den Einfluss wichtiger Variablen (sog. Störvariablen) zu erkennen, deren Einfluss zu bestimmen und ggf. auszuschalten. So sollten in unserer Studie die wichtigsten Einflussfaktoren in Betracht gezogen werden. Wir überprüften deshalb, inwieweit

- die demographischen Angaben (wie Alter, Geschlecht, Schulbildung und Beruf),

- die klinischen Parameter (AHI, BMI, AH-Dauer, $\mathrm{S}_{\mathrm{aO}_{2}}$ ),

- die fahrtechnischen Angaben (wie Sehfähigkeit, Motorik, Rolle der Fahrpraxis) sowie

- die selbstanamnestischen Angaben zur OSAS-Problematik (körperliche und geistige Leistungsfähigkeit, Tagesschläfrigkeit $[22,23]$ und Unfallhäufigkeit)

mit der Fehlerrate beim Test (Spurabweichungen beim tracking) korrelieren. 


\section{Methodik}

Messinstrumente

Fahrsimulator „carsim“ zur Erfassung der Daueraufmerksamkeit

Der von uns entwickelte Fahrsimulatortest „carsim“ ist ein menuegesteuertes Turbo-Pascal-Programm, das auf einem handelsüblichen PC betrieben werden kann.

Bei diesem Test wird das Bild einer Straße mit Randbegrenzung und Mittelstreifen polychrom simuliert (Abb. 2). An der rechten Straßenseite werden Hindernisse in Form von Durchfahrtsverbotsschildern präsentiert, die jeweils nur kurz sichtbar sind $(200 \mathrm{~ms})$. Ihr Erscheinen erfolgt zeitlich zufällig, wobei in einem 5-Minuten-Abschnitt 18 Ereignisse dargeboten werden (Standardeinstellung: vgl. Tab. 2).

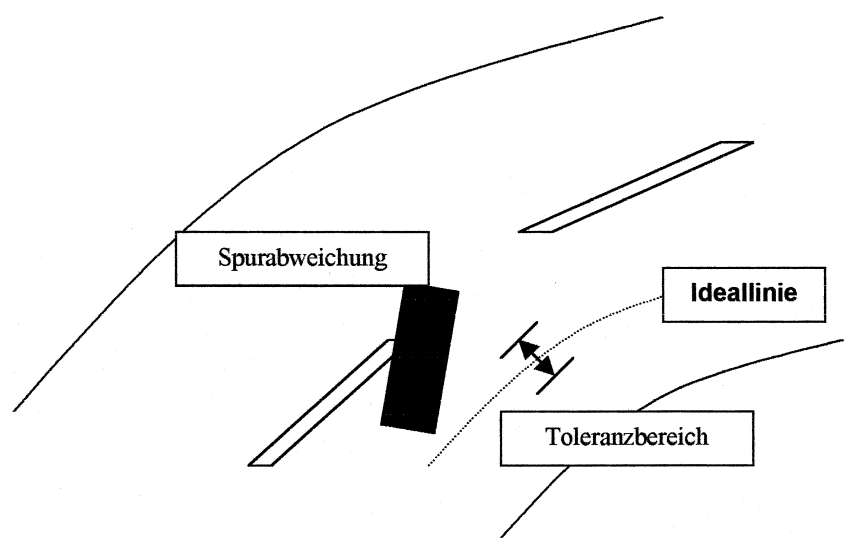

Abb. 2 Fahrsimulator „carsim“ (Büttner et al. 1999).

Tab. 2 Standardeinstellung von carsim

\begin{tabular}{ll}
\hline Testparameter & Standardeinstellung \\
\hline Testlänge & $30 \mathrm{~min}$ (6 Sektionen à $5 \mathrm{~min}$ ) \\
Übungsphase & $5 \mathrm{~min}$ \\
Geschwindigkeit & $100 \mathrm{~km} / \mathrm{h}$ \\
Anzahl der Kurven & 1 je Sektion \\
Anzahl der Hindernisse & 3 je Sektion \\
Sichtbarkeit der Hindernisse & $200 \mathrm{~ms}$ \\
\hline \hline
\end{tabular}

Der Patient bzw. Proband hat nun z.B. die Aufgabe, mit Hilfe eines Lenkrades auf seiner Fahrbahn die Idealspur zu halten (tracking) und anhand zweier Knöpfe (beide gleiche Funktion), die sich an der Lenkradkonsole befinden, auf die auftauchenden Hindernisse zu reagieren (visual search). Abhängig von den Lenkradbewegungen wird die Position des Fahrzeugs auf der Straße jeweils neu berechnet und on-line visualisiert.

Das Programm registriert u.a. die Abweichungen von der Spur (tracking), die Anzahl der richtigen Reaktionen (visual search) sowie die Reaktionszeit $[5,6]$.
Patientenbogen zur Erfassung allgemeiner Angaben

Für die Erhebung der demographischen, klinischen, fahrtechnischen und schlafbezogenen Angaben wurde ein spezieller Fragebogen entwickelt.

Der demographische Teil umfasst u.a. Fragen zu Alter, Geschlecht, Schulbildung sowie den Beruf des jeweiligen Patienten oder Probanden.

Der klinische Teil erfasst den Bodymass-Index (BMI), die Störungsdauer, den Apnoe-Hypopnoe-Index (AHI), die tiefste Sauerstoffentsättigung sowie die längste Apnoe-Pause. Er beinhaltet somit den zur Diagnose nach ICD-9 notwendigen medizinischen Status (erhoben anhand durchgeführter Eingangsuntersuchung und erfolgter Polysomnographie).

Die Daten bez. Fahrtüchtigkeit erfragen die subjektiven Beeinträchtigungen des Studienteilnehmers hinsichtlich seiner Sehfähigkeit und seiner Motorik (u.a. Störungen der Bewegungskoordination z.B. Zittern, Schwäche oder Lähmungserscheinungen der oberen Extremitäten). Diese Angaben sind insbesondere für eine Studienteilnahme erforderlich, da sowohl visuelle als auch motorische Beeinträchtigungen die Ergebnisse am Fahrsimulator verfälschen könnten. Außerdem wurde der Betreffende befragt, wie er seine Fahrpraxis anhand von 2 Kategorien einschätzt (kein Führerschein oder wenig Fahrpraxis bzw. ausreichend oder viel Fahrpraxis).

Der letzte Teil umfasst die subjektiven schlafbezogenen Angaben. In ihm beschreiben die Studienteilnehmer selbstanamnestisch eventuelle Erkrankungssymptome sowie deren eingeschätzte Relevanz (z. B. bezüglich körperlicher und geistiger Leistungsfähigkeit, Tagesmüdigkeit und Unfallhäufigkeit).

Epworth Sleepiness Scale (ESS) zur Erhebung der subjektiven Schlafqualität

Die Epworth Sleepiness Scale [22,23] erhebt anhand einer Selbstbeurteilung die Wahrscheinlichkeit des Einnickens in bestimmten Situationen tagsüber und ist insbesondere bei OSAS- und Narkolepsie-Patienten geeignet.

Der Fragebogen kann sowohl in Einzel- wie auch in Gruppenversuchen verwendet werden, wobei die Einzelerhebung der Gruppenerhebung vorzuziehen ist, um unpersönliche Testsituationen zu vermeiden. Die Fragen sollen in einer angenehmen Räumlichkeit und ohne Zeitdruck beantwortet werden. Im Durchschnitt beträgt die Bearbeitungszeit ein bis zwei Minuten.

Die ESS umfasst acht Fragen bzw. Items zur Tagesmüdigkeit (bspw. im Sitzen, beim Fernsehen oder beim Autofahren). Die Gewichtung der Antworten erfolgt anhand codierter Antwortkategorien, deren Beurteilung mit einem vierstufigen Häufigkeitsurteil (Skala von 0 bis 3). Die gewichteten Itemantworten werden dann zu einem Summenwert addiert.

Item- und Faktoranalysen ergaben bei der ESS eine sehr gute Interne Konsistenz (Cronbachs alpha =0,88) und eine hohe Retest-Reliabilität nach fünf Monaten bei Normalpersonen $(\mathrm{rtt}=0,822 ; \mathrm{n}=87 ; \mathrm{p}<0,001)$. 


\section{Studiendesign}

Die Studienteilnehmer sollten, je nach Fragestellung, den Fahrsimulatortest ein- bis zweimal 30 bzw. 45 Minuten lang absolvieren (vgl. Standardeinstellung: Tab. 2).

Die Testung mittels Fahrsimulator erfolgte nachmittags. Sie fand in einem schallisolierten, unbeleuchteten und abgedunkelten Raum (einzige Lichtquelle: Computerbildschirm) statt. Die Testinstruktion war standardisiert und wurde immer durch denselben Versuchsleiter gegeben.

Die Studienteilnehmer (Patienten, Probanden) durften 4-6 Stunden vor der Untersuchung weder Alkohol noch coffeinhaltige Getränke zu sich genommen haben. Sie sollten nicht unter einem Hunger- oder Durstgefühl leiden. Außerdem durften sie keine aufmerksamkeitsbeeinträchtigenden Medikamente eingenommen haben.

Zusätzlich wurden alle Teilnehmer mittels Fragebogen, bestehend aus einem selbst entwickelten Patientenbogen und der Epworth Sleepiness Scale (ESS), bez. ihrer Tagesschläfrigkeit überprüft. Probanden mit einem Epworth-Score $\geq 9$ wurden aus der Studie ausgeschlossen. In dem Patientenbogen wurden alle klinischen Daten (Apnoe-Hypopnoe-Index [AHI], tiefste Sauerstoffsättigung und längste Apnoe mittels Polysomnographie [PSG], Bodymass-Index [BMI], Krankheitsdauer) erfasst, die demographischen Parameter (Alter, Geschlecht, Schul-, Berufsausbildung) sowie Angaben zur Fahrpraxis und Unfallhäufigkeit erfragt.

\section{Patienten}

Die Untersuchung erfolgte an drei unabhängigen Gruppen (Gruppe 1: Gesunde; Gruppe 2: OSAS-Patienten unter nCPAP; Gruppe 3: unbehandelte OSAS-Patienten, siehe Tab. 3).

100 Probanden (90 Männer und 10 Frauen) wurden per Zufallsprinzip in einem Betriebsarztzentrum ausgewählt. 30 Patienten (29 Männer und 1 Frau) standen unter nCPAPDauertherapie (> 6 Monate). 200 OSAS-Patienten (165 Männer und 35 Frauen) wurden anhand ihrer klinischen Diagnose (ICSD 780.53-0; AHI $\geq 10$ ) rekrutiert. Bei den Patienten wurde dann eine komplette Polysomnographie nach den Kriterien der DGSM [31] zur Diagnosestellung und/oder -sicherung durchgeführt.

Zusätzlich erfolgte bei allen Studienteilnehmern eine psychiatrische/neurologische Untersuchung, bei den Patienten während der stationären Aufnahme, bei den Probanden hingegen während einer arbeitsmedizinischen Untersuchung.

Demographische und klinische Daten (siehe Tab.3): Beim Vergleich der Mittelwerte der anamnestischen Angaben zur Symptomatik mittels ESS unterschieden sich die Gruppen höchst signifikant von einander $(p<0,001)$. Die Patienten unter CPAP-Dauertherapie lagen hinsichtlich des ESS-Scores zwischen den Gesunden und den unbehandelten OSASPatienten. Beim AHI unterschieden sich die Patienten unter nCPAP höchst signifikant $(\mathrm{p}<0,001)$ von den unbehandelten OSAS-Patienten. Der BMI lag bei den Probanden tiefer als bei den OSAS-Patienten und den Patienten unter nCPAP $(\mathrm{p}=0,001)$. In der Schul- bzw. Berufsausbildung unterschieden sich die Gruppen nicht, der größte Teil der Studienteilnehmer hatte die 10. Klasse absolviert und eine abgeschlossene Berufsausbildung. Bei der Störungsdauer gaben die Patienten unter nCPAP-Therapie ( $>6$ Monate) längere Zeiten als die bislang unbehandelten an $(\mathrm{p}<0,001)$. Bezüglich ihrer Fahrpraxis $(\mathrm{p}=0,248)$ unterschieden sich Gesunde, behandelte und unbehandelte Patienten nicht, wohl aber in der Angabe ihrer Unfallhäufigkeit $(p=0,007)$. Gesunde gaben durchschnittlich mehr Verkehrunfälle als OSAS-Patienten und Patienten unter nCPAP an (Tab.4).

\section{Statistik}

Die Daten wurden mit dem Edit-Programm von DOS entsprechend der Fragenbogen und der erhaltenen Testbefunde in mehrere Einzeldateien eingegeben, deren Verknüpfung die Versuchspersonen-Nummer (Vp-Nr.) darstellte. Die Rohdaten wurden codiert, in eine Gesamtdatei übertragen und für das Statistikprogramm SPSS transformiert. Die Auswertung wurde dann mit Hilfe des SPSS-Programmes ausgeführt.

Nach einer Beschreibung der Untersuchungspopulation hinsichtlich demographischer, klinischer, fahrtechnischer und schlafbezogener Daten erfolgte die Überprüfung der Stichproben auf Normalverteilung. Diese wurde mit dem Kolmogorov-

Tab. 3 Demographische und klinische Daten der Studienteilnehmer

\begin{tabular}{lcccc}
\hline Gruppe & KG1: Gesunde & KG2: OSAS unter nCPAP & EGs: OSAS-Patienten & Signifikanz ( $p=$ ) \\
\hline Anzahl der Vp & 100 & 30 & 200 & 0,274 \\
Altersmittelwerte & $34,2 \pm 10,2$ & $56,0 \pm 7,1$ & $55,2 \pm 12,1$ & 0,976 \\
BMI & $26,4 \pm 4,5$ & $30,9 \pm 7,0$ & $30,9 \pm 4,8$ & 0,892 \\
ESS & $5,5 \pm 3,1$ & $9,7 \pm 4,8$ & $11,4 \pm 4,8$ & 0,884 \\
Störungsdauer & & $5,4 \pm 6,3$ & $1,9 \pm 2,7$ & 0,904 \\
AHI & & $2,3 \pm 8,4$ & $32,4 \pm 19,5$ & 0,923 \\
tiefste $\mathrm{O}_{2}$-Sättigung & $0,6 \pm 0,9$ & $90,3 \pm 13,0$ & $82,4 \pm 9,4$ & 0,771 \\
Unfälle & $0,3 \pm 0,8$ & $0,3 \pm 0,8$ &
\end{tabular}

Gruppen (EG = Experimentalgruppe; KG=Kontrollgruppen); EG=OSAS-Patienten vor Therapieeinleitung; KG1=gesunde Probanden; KG2 = OSAS-Patienten unter nCPAP-Dauertherapie > 6 Monate; $V p=$ Versuchspersonen; BMI=Bodymass-Index; ESS =Epworth Score; Störungsdauer=Zeit, seit der Schlafapnoe besteht (subjektive Angabe); $\mathrm{AHI}=$ Apnoe-Hypopnoe-Index; $\mathrm{O}_{2}=$ Sauerstoff; Unfall=Unfallhäufigkeit in den letzten 5 Jahren (subjektive Angabe); Signifikanz ( $\mathrm{p}=$ ) = Signifikanz der demographischen und klinischen Daten bez. ihres Störeinflusses auf die Spurabweichungen 
Tab. 4 Spurabweichungen mit und ohne Fahrpraxis bzw. Führerschein

\begin{tabular}{llll}
\hline Gruppe & KG1: Gesunde & KG2: OSAS unter nCPAP & EGs: OSAS-Patienten \\
\hline Spurabweichungen mit Fahrpraxis & $22,1 \pm 43,9(n=91)$ & $1603,8 \pm 6369,7(n=29)$ & $2111,3 \pm 6564,5(n=178)$ \\
Spurabweichungen ohne Fahrpraxis & $31,6 \pm 52,5(n=9)$ & $0,0 \pm 0,0(n=1)$ & $8057,9 \pm 10877,9(n=22)$ \\
Signifikanz & $p=0,545$ & & $p<0,001$
\end{tabular}

Gruppen (EG = Experimentalgruppe; KG = Kontrollgruppen); EG = OSAS-Patienten vor Therapieeinleitung; KG1 = gesunde Probanden; KG2 = OSAS-Patienten unter nCPAP-Dauertherapie $>6$ Monate

Smirnov-Test durchgeführt. Die Überprüfung parametrisch vs. nonparametrisch ergab eine Normalverteilung der Gruppen hinsichtlich Alter und Geschlecht, d.h. die Untersuchungspopulation ist repräsentativ.

Im ersten Teil der Untersuchung erfolgte nun die Normwerteerstellung anhand des gesunden Vergleichskollektives sowie die Überprüfung des Fahrsimulatortests „carsim“ hinsichtlich seiner Gütekriterien [7].

Zur Überprüfung des Einflusses der wichtigen klinischen und demographischen Variablen wurde im weiteren Verlauf der Zusammenhang zwischen der Diagnose, der Schlafqualität (klinische und anamnestische Parameter) und den gemessenen Daueraufmerksamkeitswerten (Spurabweichungen bei „carsim“) anhand von Korrelationen sowie einer univariaten, einfaktoriellen Varianzanalyse mit Kovarianzen kontrolliert. Die Homogenität der Varianzen wurde hierbei mit dem Bartlett-Test überprüft.

Als unabhängige Variable wird die Gruppeneinteilung anhand der Diagnosestellung (nach ICSD und Polysomnographie) verwendet.

Die Kovarianzen der Studie setzten sich aus den verschiedenen demographischen Angaben (wie Alter, Geschlecht, Schul- bildung, Beruf), klinischen Parametern (bspw. Epworth Score, Bodymass-Index, Apnoe-Hypopnoe-Index, längste Apnoe, tiefste $\mathrm{O}_{2}$-Sättigung), fahrtechnischen Angaben (wie Sehfähigkeit, motorische Funktionsfähigkeit, Fahrerlaubnis, Fahrpraxis) sowie subjektiven schlafbezogenen Angaben (z.B. Tagesmüdigkeit, körperliche und geistige Leistungsfähigkeit, Unfallhäufigkeit, Einnicken am Steuer) zusammen.

Zu den abhängigen Variablen der Untersuchung zählen alle gemessenen Komponenten der Daueraufmerksamkeit. insbesondere die Abweichungen von der Spur.

\section{Ergebnisse}

Die Fahrpraxis beeinflusste die tracking-Leistung nicht, d.h. gesunde Personen mit Fahrpraxis schnitten beim tracking nicht besser $a b$ als Personen ohne Fahrpraxis $(p=0,545)$. Im Gegensatz dazu fand sich bei den unbehandelten OSASPatienten ein höchst signifikanter Unterschied $(p<0,001)$ zwischen Patienten mit und ohne Fahrpraxis. OSAS-Patienten ohne Führerschein wiesen $\mathrm{x}=8058 \pm 10878$ Spurabweichungen, Patienten mit Führerschein $\mathrm{x}=2111 \pm 6564$ Spurabweichungen auf (Tab. 4, Abb. 3).

Die demographischen (Alter, Geschlecht, Berufszugehörigkeit, Schulbildung), klinischen (AHI, Sauerstoffsättigung, BMI),

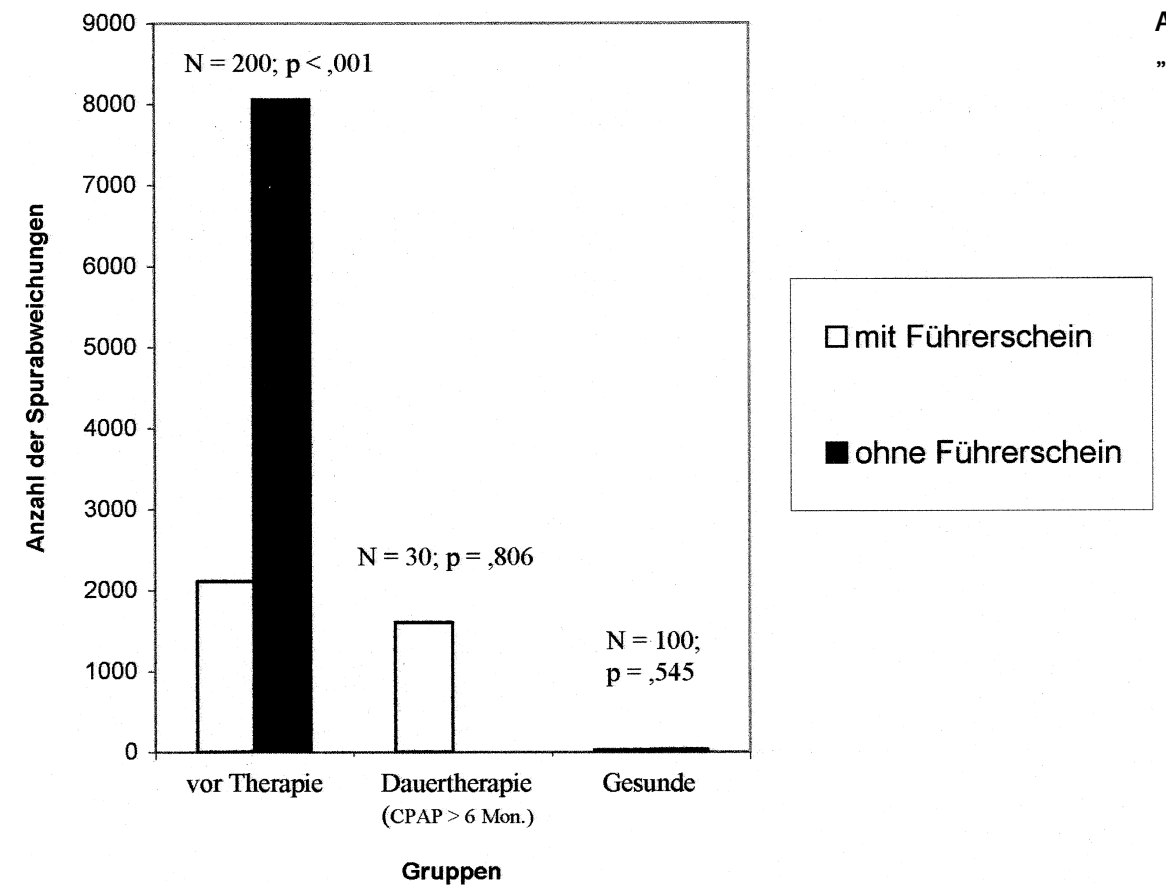

Abb. 3 Rolle der Fahrpraxis am Fahrsimulator "carsim“. 
fahrtechnischen (Sehfähigkeit, motorische Funktionsfähigkeit) sowie selbstanamnestischen Parameter (körperliche und geistige Leistungsfähigkeit, Tagesmüdigkeit, Unfallhäufigkeit) übten keinen signifikanten Einfluss auf die Fehlerrate beim tracking aus (Tab. 3 ).

\section{Diskussion}

Nur bei unbehandelten OSAS-Patienten spielte die Fahrpraxis bei der tracking-Fehlerrate eine relevante Rolle, d.h. Personen ohne Fahrpraxis schnitten hier schlechter ab. Da sich dieser Unterschied aber weder bei Gesunden noch bei Patienten unter nCPAP-Dauertherapie (>6 Monate) nachweisen ließ, muss davon ausgegangen werden, dass mangelhafte Fahrpraxis bei unbehandelten OSAS-Patienten eine Störvariable für den Fahrsimulator „carsim“ darstellt. Unbehandelte OSASPatienten scheinen demzufolge nicht nur extrem in ihrer Aufmerksamkeit eingeschränkt zu sein, sondern es fehlen ihnen auch wichtige Coping-Strategien, um Defizite in der Fahrpraxis ausgleichen zu können. Bei den bisher durchgeführten Studien zum Zusammenhang zwischen Unfallhäufigkeit und Tagesschläfrigkeit/Aufmerksamkeits-Tests wurde die Rolle der Fahrpraxis nicht berücksichtigt.

Abweichend von dem in unserer Studie erhaltenen Befund kein Zusammenhang zwischen Unfallhäufigkeit und Testergebnis - fanden sowohl Findley $[11,12,13]$ als auch Randerath [32] einen kausalen Zusammenhang zwischen den beiden Parametern. So wies Findley anhand von Daten der Unfallbehörde Virginias/USA einen Zusammenhang zwischen Unfallhäufigkeit und Schlafapnoesyndrom sowie eine Abhängigkeit vom Schweregrad der Erkrankung nach. Weiterhin fand er eine Korrelation zwischen der Anzahl der Unfälle und der Fehlerrate beim Fahrsimulatortest „Stear Clear“. Bei Randerath wiesen Personen, die mehr als zwei Unfälle in den letzten zwei Jahren angaben, einen signifikant höheren Fehlerwert im Vigilanztest auf. Dieses Ergebnis stimmt auch mit einer Patientenbefragung durch Cassel überein, bei der ein großer Teil der Patienten Verkehrsunfälle auf überhöhte Tagesmüdigkeit zurückführte $[7,8]$. Krieger et al. konnte nachweisen, dass sowohl die Unfallrate als auch Beinaheunfälle unter nCPAPTherapie reduziert werden konnten [24].

Da das von uns gefundene abweichende Ergebnis ebenfalls auf eine Patientenbefragung zurückgeht (objektive Daten sind aufgrund der bestehenden Gesetzeslage in Deutschland nicht zu erhalten), stellt sich die Frage, inwieweit die Patienten bereit sind, derart brisante Fragestellungen korrekt zu beantworten oder ob Bedenken hinsichtlich möglicher Konsequenzen, insbesondere bei Berufskraftfahrern und aufgrund der seit Jahresbeginn verschärften gesetzlichen Regelungen für Mitarbeiter im Fahrdienst, die Angaben beeinflusst.

Die tracking-Komponente scheint nur geringfügig für Störvariable anfällig zu sein. So zeigten sich weder alters- noch bildungsbedingte Einflüsse. Ebenso erwiesen sich die klinischen Parameter (AHI, BMI) nicht als Störvariable. Auch die Überprüfung der anamnestischen Angaben (ESS) sowie die Unfallhäufigkeit übten keinerlei Einfluss auf die Durchführung bzw. die Ergebnisse des Tests aus. Dieses Ergebnis deckt sich sowohl mit den Befunden von George [14], der keinen signifikanten Zusammenhang zwischen AHI und den Ergebnissen am Fahrsimulator „DADT“ (Divided-Attention-Driving-
Test) feststellen konnte, als auch mit denen von Randerath [32].

Zusammenfassend lässt sich daher sagen, dass klinische und demographische Größen Fahrsimulationsprozesse nicht beeinflussen. Allerdings sollte die Rolle der Fahrpraxis bei unbehandelten Patienten beachtet werden, so dass bei Personen ohne Fahrpraxis ein anderes Testverfahren angewendet werden muss.

\section{Literatur}

${ }^{1}$ Aldrich MS. Sleep disorders. Current Opinion in Neurology and Neurosurgery 1992; 5: 240-246

2 American Thoracic Society (ATS). Sleep apnea, sleepiness and driving risk. Am J Respir Crit Care Med 1994; 150: 1464 - 1473

${ }^{3}$ Bédard MA, Montplaisir J, Richer F, Malo J. Nocturnal hypoxemia as a determinant of vigilance impairment in sleep apnea syndrome. Chest 1991; 100: $367-370$

${ }^{4}$ Bradley TD, Rutherford R, Grossman RF, Lue F, Zamel N, Moldofsky $\mathrm{H}$, Phillipson EA. Role of daytime hypoxemia in the pathogenesis of right heart failure in the obstructive sleep apnea syndrome. Am Rev Respir Dis 1985; 131: 835-839

${ }^{5}$ Büttner A, Randerath W, Rühle KH. Fahrsimulatorvalidierung. Somnologie 1999a; 3 (Suppl. 1): 9-10

${ }^{6}$ Büttner A, Randerath W, Rühle KH. Therapieverlaufskontrolle der Daueraufmerksamkeit anhand eines neuen Fahrsimulatortests bei OSAS-Patienten. Somnologie 1999b; 3 (Suppl. 1): 10

${ }^{7}$ Büttner A, Randerath W, Rühle KH. Normwerte und Gütekriterien eines interaktiven Fahrsimulators („carsim“). Zur Veröffentl. vorgesehen. 2000

${ }^{8}$ Carscardon MJ, Dement WC, Mitler MM, Roth T, Westbrook PR, Keenan S. Guidelines for the multiple sleep latency test (MSLT): a standard measure of sleepiness. Sleep 1986; 9: 519-524

${ }^{9}$ Cassel W, Ploch T. Sleep apnea accidents: Health risk for healthy people? In: Peter JH, Penzel T, Podszus T, von Wichert P (Hrsg). Sleep and Health Risk. Berlin, Heidelberg, New York: Springer $1991 ; 279-285$

${ }^{10}$ Cassel W, Ploch T, Becker C, Dugnus D, Peter JH, von Wichert P. Risk of traffic accidents in patients with sleep-disordered breathing: reduction with nasal CPAP. Eur Respir J 1996; 9: 2606-2611

${ }^{11}$ Findley LJ, Unverzagt ME, Suratt PM. Automobile accidents involving patients with obstructive sleep apnea. Am Rev Respir Dis 1988; 138: $337-340$

${ }^{12}$ Findley LJ, Fabrizio MJ, Knight H, Norcross BB, Laforte AJ, Suratt PM. Driving simulator performance in patients with sleep apnea. Am Rev Respir Dis 1989; 140: 529-530

${ }^{13}$ Findley LJ. Automobile driving in sleep apnea, in: Sleep and Respiration. Wiley-Liss, Inc 1990; 337 - 345

${ }^{14}$ Findley LJ, Weiss WJ, Jabour ER. Drivers with untreated sleep apnea: a cause of death and serious injury. Arch Intern Med 1991; 151: $1451-1452$

${ }^{15}$ Findley LJ, Unverzagt M, Guchu R, Fabrizio M, Buckner J, Suratt P. Vigilance and automobile accidents in patients with sleep apnea or narcolepsy. Chest 1995; 108: 619-624

${ }^{16}$ George CFP, Boudreau AC, Smiley A. Simulated driving performance in patients with obstructive sleep apnea. Am J Respir Crit Care Med 1996a; 154: 175-181

${ }^{17}$ George CFP, Boudreau AC, Smiley A. Comparison of simulated driving performance in narcolepsy and sleep apnea patients. Sleep 1996b; 19(9): 711 - 717

${ }^{18}$ George CFP, Boudreau AC, Smiley A. Effects of nasal CPAP on simulated driving performance in patients with obstructive sleep apnea. Thorax 1997; 52: 648-653 
${ }^{19}$ Gerdesmeyer C, Randerath W, Rühle KH. Zeitliche Abhängigkeit der Fehlerzahl bei Messung der Daueraufmerksamkeit mittels Fahrsimulator vor und nach nCPAP-Therapie bei Schlafapnoesyndrom. Somnologie 1997; 1: 165 - 170

${ }^{20} \mathrm{He}$ J, Kryger MH, Zorick FJ, Conway W, Roth Th. Mortality and apnea index in obstructive sleep apnea. Experience in 385 male patients. Chest 1988; 94: 9-14

${ }^{21}$ Hoddes E, Zarcone V, Smythe H, Phillipps R, Dement WC. Quantification of sleepiness: a new approach. Psychophysiology 1973; 10: $431-436$

${ }^{22}$ Johns MW. A new method of measuring daytime sleepiness: the Epworth sleepiness scale. Sleep 1991; 14: 540-545

${ }^{23}$ Johns MW et al. Daytime Sleepiness, Scoring and Obstructive Sleep Apnea, The Epworth Sleepiness Scale. Chest 1993; 103: 30-36

${ }^{24}$ Kribbs NB, Getsy JE, Dinges DF. Investigation and management of daytime sleepiness in sleep apnea. In: Saunder NA, Sullivan CE (Hrsg). Sleeping and Breathing 2. New York: Marcel Dekker, 1993a: $575-604$

${ }^{25}$ Kribbs NB, Pack AL, Kline LR, Getsy JE, Schuett JS, Henry JN, Maislin G, Dinges DF. Effects of one night without nasal CPAP treatment on sleep and sleepiness in patients with obstructive sleep apnea. Am Rev Despir Dis 1993b; 147: 1162 - 1168

${ }^{26}$ Krieger J, Meslier N, Lebrun T, Levy P, Phillip-Joet F, Sailly JC, Racineux JJ. Accidents in obstructive sleep apnea patients treated with nasal continuous positive airway pressure. A prospective study. Chest 1997; 112: 1561 - 1566

${ }^{27}$ Lamphere J, Roehrs T, Wittig R, Zorick F, Conway WA, Roth T. Recovery of alertness after CPAP in apnea. Chest 1989; 96: $1364-1367$

${ }^{28}$ Martin SE, Engleman HM, Deary IJ, Douglas NJ. The effect of sleep fragmentation on daytime function. Am J Respir Crit Care Med 1996; 153: 1328 - 1332

${ }^{29}$ Mitler M, Carskadon M, Czeiler C, Dement W, Dinges D, Graeber R. Catastrophes, Sleep and public policy: consensus report. Sleep 1988; 11: 100-109

${ }^{30}$ Moscowitz H, Burns M. The effects of alcohol and valium, singly and in combination, upon driving-related skills performance. Proceedings of the $21^{\text {st }}$ Conference of the Association for Automobile Medicine. Vancouver, BC, 15-17. September 1977: $226-240$

${ }^{31}$ Penzel T, Hajak G, Hoffmann RM, Lund R, Podzus T, Pollmächer T, Schäfer T, Schulz H, Sonnenschein W, Spieweg I. Empfehlungen zur Durchführung und Auswertung polygraphischer Ableitungen im Schlaflabor. Z EEG-EMG 1993; 24: 65 - 70

32 Poceta JSt, Timms RM, Jeong DU, Swui-Ling H, Erman MK, Mitler MM. Maintenance of wakefulness test in obstructive sleep apnea syndrome. Chest 1992; 101: 893-897

33 Podszus T, Bauer W, Mayer J, Penzel T, Peter JH, von Wichert P. Sleep apnea and pulmonary hypertension. Klin Wochenschr 1986; 64: $131-134$

${ }^{34}$ Randerath W, Gerdesmeyer C, Ströhlein G, Rühle K-H. Messung der Vigilanz mittels Fahrsimulator vor und nach nCPAP Vergleich zweier Simulationsprogramme mit unterschiedlicher Ereignishäufigkeit. Somnologie 1997; 1: 110-114

${ }^{35}$ Randerath W, Siller C, Gil G, Rühle KH. Fahrsimulatortest zur Erfassung der Daueraufmerksamkeit - Untersuchung bei Normalpersonen und bei Patienten mit obstruktiven Schlafapnoesyndrom vor und nach Therapie. zur Veröffentl. vorgesehen. 1998

${ }^{36}$ Roehrs T, Zoricks F, Wittig R, Conway W, Roth T. Predictors of objective level of daytime sleepiness in patients with sleeprelated breathing disorders. Chest 1989; 95: $1202-1206$

${ }^{37}$ Walsleben JA. The measurement of daytime wakefulness. Chest 1992; 101: 890-891
${ }^{38}$ Weeß HG. Schläfrigkeit und sozialmedizinisches Risiko. Theorethische Grundlagen. Arbeitspapier des Schlaflabors Pfalzklinik Landeck, 30.1.1997

39 Weeß HG, Land R, Gresele C, Böhning W, Sauter C, Steinberg RAG Vigilanz der DGSM. Vigilanz, Einschlafneigung, Daueraufmerksamkeit, Müdigkeit, Schläfrigkeit. Die Messung müdigkeitsbezogener Prozesse bei Hypersomnien - Theoretische Grundlagen. Somnologie 1998; 2: $32-34$

\section{A. Büttner \\ Am Rain 24 \\ 35039 Marburg}

